\title{
Identifying IncRNA-mediated regulatory modules via ChIA-PET network analysis
}

\author{
Denise Thiel ${ }^{1}$, Nataša Djurdjevac Conrad ${ }^{3}$, Evgenia Ntini ${ }^{1}{ }^{2}$, Ria X. Peschutter $^{1}$, Heike Siebert $^{2}$ \\ and Annalisa Marsico 1,2,4*
}

\begin{abstract}
Background: Although several studies have provided insights into the role of long non-coding RNAs (IncRNAs), the majority of them have unknown function. Recent evidence has shown the importance of both IncRNAs and chromatin interactions in transcriptional regulation. Although network-based methods, mainly exploiting gene-IncRNA co-expression, have been applied to characterize IncRNA of unknown function by means of 'guilt-by-association', no strategy exists so far which identifies mRNA-IncRNA functional modules based on the 3D chromatin interaction graph.

Results: To better understand the function of chromatin interactions in the context of IncRNA-mediated gene regulation, we have developed a multi-step graph analysis approach to examine the RNA polymerase II ChIA-PET chromatin interaction network in the K562 human cell line. We have annotated the network with gene and IncRNA coordinates, and chromatin states from the ENCODE project. We used centrality measures, as well as an adaptation of our previously developed Markov State Models (MSM) clustering method, to gain a better understanding of IncRNAs in transcriptional regulation. The novelty of our approach resides in the detection of fuzzy regulatory modules based on network properties and their optimization based on co-expression analysis between genes and gene-IncRNA pairs. This results in our method returning more bona fide regulatory modules than other state-of-the art approaches for clustering on graphs.

Conclusions: Interestingly, we find that IncRNA network hubs tend to be significantly enriched in evolutionary conserved IncRNAs and enhancer-like functions. We validated regulatory functions for well known IncRNAs, such as MALAT1 and the enhancer-like IncRNA FALEC. In addition, by investigating the modular structure of bigger components we mine putative regulatory functions for uncharacterized IncRNAs.
\end{abstract}

Keywords: IncRNA, Modules, Network analysis, ChIA-PET, Gene regulation

\section{Introduction}

Long non-coding RNAs (lncRNAs), an heterogeneous group of non-coding transcripts longer than 200 nucleotides, are expressed in a time- and tissuespecific fashion and have been shown to regulate cellular processes such as development, imprinting, Xchromosome inactivation, cancer and immunity $[1,2]$. The discovery of extensive transcription of these noncoding transcripts provides an important new perspective on the centrality of RNAs in gene regulation [3]. To date,

\footnotetext{
*Correspondence: marsico@molgen.mpg.de

${ }^{1}$ Max Planck Institute for Molecular Genetics, Berlin, Ihnestraße 63-73, 14195 Berlin, Germany

2Department of Mathematics and Informatics, Freie Universität, Berlin, Arnimallee 7, 14195 Berlin, Germany

Full list of author information is available at the end of the article
}

next-generation sequencing data generated by several consortia, such as the ENCODE [4] or FANTOM5 [3] leads to an estimate of the number of potential lncRNA transcripts of about 20000. Although only a smaller fraction of such transcripts might be functional, and despite the substantial progress in mapping lncRNAs, the detailed functional mechanisms for most of them remain elusive [2]. The gap in the understanding of the functional roles of the lncRNAs has largely been due to their poor evolutionary conservation, but also to the limited ability of tools to characterize lncRNA interactions with either proteins, DNA and RNA on a large scale. Concomitant with the increasing number of lncRNAs, a number of resources collecting and curating functional information about lncRNAs have been built in recent years [5-8].

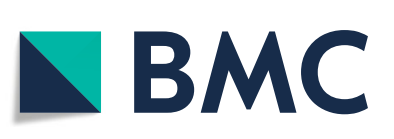

(c) The Author(s). 2019 Open Access This article is distributed under the terms of the Creative Commons Attribution 4.0 International License (http://creativecommons.org/licenses/by/4.0/), which permits unrestricted use, distribution, and reproduction in any medium, provided you give appropriate credit to the original author(s) and the source, provide a link to the Creative Commons license, and indicate if changes were made. The Creative Commons Public Domain Dedication waiver (http://creativecommons.org/publicdomain/zero/1.0/) applies to the data made available in this article, unless otherwise stated. 
It has been shown, among others, that lncRNAs can regulate the expression either of their neighboring genes in cis, or of more distant genes in trans. LncRNAs may function via binding to RNA Binding Proteins (RBPs), such as chromatin regulators that can bind both RNA and DNA, or by interactions with other nucleic acids [9].

A major category of well-studied functional lncRNAs is those implicated in coordinated gene silencing, either in cis (e.g. the lncRNA Xist, involved in X-chromosome inactivation) or in trans (e.g. HOTAIR). Both XIST and HOTAIR have been shown to mediate epigenetic mechanisms of gene silencing $[10,11]$.

Genome-scale mapping of histone modifications and enhancer-binding proteins has helped to identify lncRNAs involved in gene activation. Enhancers are regulatory sequences that can activate gene expression, and their function depends on the interplay between DNA sequences, DNA-binding proteins, and architecture [12]. In the last five years, the functional landscape of enhancers has become more complex with the evidence that active enhancers can transcribe structured lncRNAs. A recent study performed loss-of-function experiments and found 7 of 12 enhancer-transcribed lncRNAs affecting expression of their cognate neighboring genes [13]. More recently, HOTTIP, an enhancer-like lncRNA, has been discovered to directly interact and activate the WDR5 protein [10], a key component of the mixed lineage leukemia-Trx complex. In other cases lncRNAs activate a neighboring lncRNA, e.g., JPX regulates transcriptional activation of XIST on chromosome X [10]. Long noncoding RNAs with activating function may recruit transcriptional activators involved in the establishment of chromosome looping between the lncRNA loci and regulated promoters, such as the mediator complex [14].

The architectural landscape of the nucleus has a profound influence on gene regulation. Chromosome conformation capture technologies, such as $3 \mathrm{C}$, Hi-C, 4C, Capture-C and Chromatin Interaction Analysis by PairedEnd Tag Sequencing (ChIA-PET) have revealed elements that are distally located either on the same or separate chromosomes, to be proximal in the three dimensional nucleus [15]. The effect of such contacts, especially when they correspond to enhancer-promoter or promoter-promoter interactions, mediated by PolII or other factors, is an area of intense research [15]. There is evidence that enhancer-promoter interactions might be induced by chromatin looping and mediated by enhancerlike non-coding RNAs (ncRNAs), and that the ChIA-PET technique is suitable to detect them $[10,16]$.

Additional evidence on potential functions of lncRNAs have been obtained from methodologies which rely on expression patterns and "Guilt by Association": transcripts sharing common expression patterns are expected to be co-regulated or share common pathways $[17,18]$. Most of these methods build a coding-non-coding co-expression network, in which a node represents a molecule and an edge an expression correlation. Such a network is used to identify cellular modules involving both protein coding genes and lncRNAs, and the unknown function of lncRNAs is predicted by transferring functional annotation (e.g. Gene Ontology (GO) terms) from protein coding genes $[10,17,19]$. These approaches however detect statistical associations, and thus do not directly contribute to an understanding of detailed mechanisms of lncRNAmediated gene regulation.

In this study we focused on lncRNA regulatory functions in the cell nucleus and constructed the chromatin interaction network involving lncRNAs, genes and other genomic regions using ChIA-PET data in the K562 cell line, which compared to $\mathrm{HiC}$ has higher genomic resolution. ChIA-PET combines ChIP with chromatin capture technology to detect interactions between genomic regions mediated by a transcription factor of interest [20]. Here, we focus on the Polymerase II (Pol II)-mediated chromatin network, as it is directly linked to transcriptional regulation. A natural representation of these data amenable to efficient analysis are complex networks, where nodes represent DNA segments or Paired-End Tags (PETs), and edges represent ChIA-PET interactions between two PETs. The analysis of chromatin interaction networks has been an area of active research in the last years, but very few studies have employed network analysis and clustering methods to study chromatin interaction networks $[15,21]$.

For many biological networks, including gene regulatory networks, the evaluation of well-established node characteristics, in particular centrality measures, are highly suitable for identification of functionally essential elements [22]. Similarly, modular organization is believed to be a generic property of such networks, allowing to uncover subnetworks responsible for a specific function. In gene regulatory networks for instance, modules often correspond to groups of interconnected cis-regulatory elements.

We developed a hierarchical network analysis approach to compute centrality properties of lncRNAs in the chromatin network, followed by a focus on the connected components of the chromosome graphs and finally reaching the level of density-based modules, that are amenable to a detailed analysis in their entirety (Fig. 1). Specifically, to identify these potential IncRNA-mediated functional modules, we implement a modified version of our previously developed Markov State Models (MSM) clustering approach [23, 24], which aims at identifying subgraphs of high connectivity. Compared to previous methods we do not rely on lncRNA-mRNA co-expression for network building, neither for clustering, but only on the topology and properties of the chromatin graph. Co-expression 


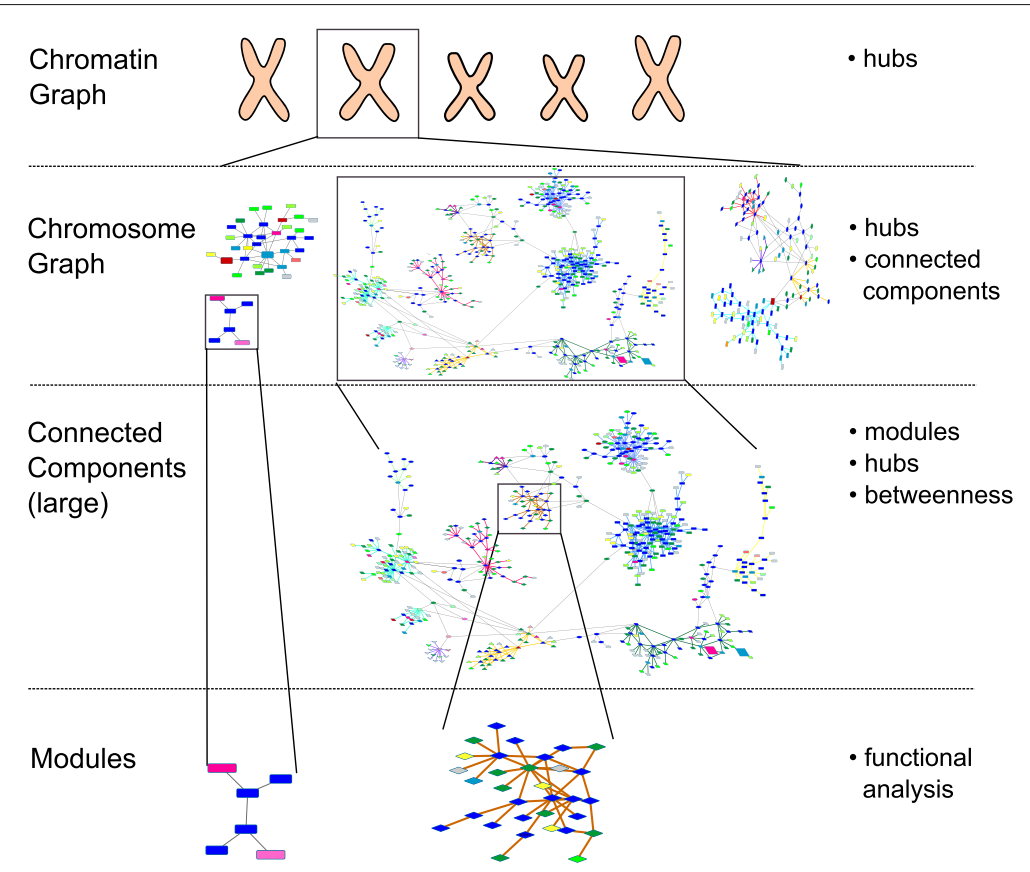

Fig. 1 Overview of the hierarchical graph analysis. The different levels represent a zoom into more detail in the graph, starting with the chromatin graph at the top, then focusing on a single chromosome followed by large connected components and lastly modules detected in the large component using the MSM algorithm or as small connected components of the corresponding chromosome. On the right, we list the different analysis steps performed at each level, focusing only on degree centrality on the level of the chromatin graph, then adding in consideration of connectivity properties as well as module detection and finally considering molecular information to assess possible functional interactions within modules. Node shapes are arbitrary and node colors symbolize different node annotations

information is incorporated only in a second step by the algorithm to fine-tune the final network partition, based on the expectation that genes and IncRNAs which are spatially coordinated and contained in the same functional module also have related expression patterns. To our knowledge, this is the first approach that defines modularity in a mRNA-lncRNA interaction network based on chromatin interactions and uses the added value of coexpression to refine interacting modules and characterize unknown regulatory RNAs.

We compare our method with other state-of-the-art graph clustering methods, and show that MSM clustering is superior in returning clusters corresponding to genuine regulatory modules, i.e. whose members exhibit a high correlation in expression between gene-gene, lncRNAgene and lncRNA-lncRNA node pairs. We evaluated our approach by matching modules and interactions to lncRNAs of known function, such as ncRNA-a3, FALEC, Xist and MALAT1 [9]. LncRNAs transcribed from enhancer regions exhibit either a high degree or high betweenness centrality, highlighting their regulatory potential in the leukemia-specific network. Finally, we inspect potential functions of lncRNA modules in big chromosome connected components, making our strategy a valuable tool towards functional annotation of lncRNAs with functions in transcriptional gene regulation.

\section{Methods}

Data collection and Pre-processing

ChIA-PET Data. The Pol II ChIA-PET interaction network in the $\mathrm{K} 562$ cell line was build based on the already processed interaction files downloaded from the ENCODE project website. Interacting pairs of genomic regions from this files corresponds to two nodes linked by an edge in our network. The data corresponding to two different ChIA-PET replicates were downloaded and only interactions supported by both replicates were retained for further analysis.

Filtering of PET interactions. As we were interested in cis long-range interactions we filtered out the $1.8 \%$ inter-chromosomal PET interactions before further analysis. Also we excluded the so-called self-ligation PETs from further analysis [25], as they represent an artifact of ChIA-PET experiments, and originate from selfcircularization ligation of the same chromatin fragment resulting in ChIA-PET sequences with both tags mapped within a short genomic distance of each other. In order to distinguish between self-ligation PETs and inter-ligations PETs, which actually correspond to two distinct interacting chromosomal regions, we performed a similar analysis to Li et al. [25]. We computed the genomic distances between PETs and plotted their frequency in each genomic bin on a log-log scale. The intersection of two 
fitted lines at $1691 \mathrm{nt}$ was taken as distance cutoff to distinguish self-ligation from inter-ligation PETs, which seem to follow two distinct power-law distributions (Fig. 2 left). Self-ligation interactions, with distances below this cutoff, were discarded from further analysis.

Expression analysis of IncRNAs and genes. Expression levels of both lncRNAs and protein-coding genes in K562 were computed from the corresponding alignment file of RNA sequencing (RNA-seq) from the Cold Spring Harbor Lab (CSHL) ENCODE track (chromatin fraction). Genomic annotation of IncRNAs and genes was taken from Gencode v24. Coordinates were lifted over the hg19 human genome assembly as all other annotations were on hg19. Read counts in protein-coding genes and lncRNAs were obtained by means of htseq-count [26] for two different replicates with default parameters (stranded, skip all reads with alignment quality lower 10, overlapping reads handled as union), using only complete gene regions (introns included) from the annotation file and converted to Reads Per Kilobase of transcript, per Million mapped reads (RPKMs). Only genes with an RPKM $>0.041$ and lncRNAs with RPKM $>0$ in both replicates or RPKM $>0.041$ in at least one replicate were considered 'detected' and retained for further analysis. The 0.041 threshold was determined by looking at the bimodal distribution of the $\log$ RPKM expression values of all genes and corresponds to the local minimum separating the two modes.

Network construction and annotation. PETs representing interacting genomic regions were annotated as 'gene', and assigned their corresponding official gene symbol if they overlapped the genomic coordinates of annotated protein-coding genes from Gencode. PETs were annotated as 'IncRNA' if they overlapped the genomic coordinates of annotated lncRNAs from Gencode. Given that the resolution of the ChIA-PET data is in the order of few kilobases, it could occur that interacting PETs might cover wide genomic regions with more than one annotated gene/IncRNA. In addition, ChIA-PET data are not strand-specific, therefore they might overlap with two or more genes/lncRNAs located on different strands. PETs corresponding to more than one gene/lncRNA location, either on the same or the opposite strand, were annotated with both gene and IncRNA names. Chromatin states in K562 from the chromHMM software genome segmentation [27] downloaded from the ENCODE website were also used to annotate interacting PETs in the network as 'enhancer', 'weak enhancer', Transcription Start Site ('TSS'), 'promoter flanking,' 'CTCF,' 'transcribed' and 'repressed' (Fig. 3b). The assignment 'repressed' was ignored because in a network containing interactions mediated by Pol II, repressed regions hold no information. It could occur that the same PET overlapped with many different features. In this case annotations were merged. For example a PET overlapping both an annotated lncRNA and an enhancer region was defined as 'IncRNA_enhancer'. If PETs did not overlap with any annotated gene, lncRNA or chromatin state, were labeled as unknown. Annotated PETs were represented as nodes in the network and an interaction between PETs as an edge. A global $(0,1)$-adjacency matrix was build to describe the overall graph, called from now on chromatin graph. The number of rows and columns of the adjacency matrix represents the number of genomic regions involved in at least one ChIA-PET interaction. A 0entry in the matrix cell corresponds to no interactions between any two PETs overlapping with these regions, while a 1-entry corresponds to a ChIA-PET interaction. A schematic view of the steps described above is given in Fig. 3b.

For gene disease annotation the disease databases OMIM [28] and DisGenet [29] were used. Disease annotation data for lncRNAs was taken from the database
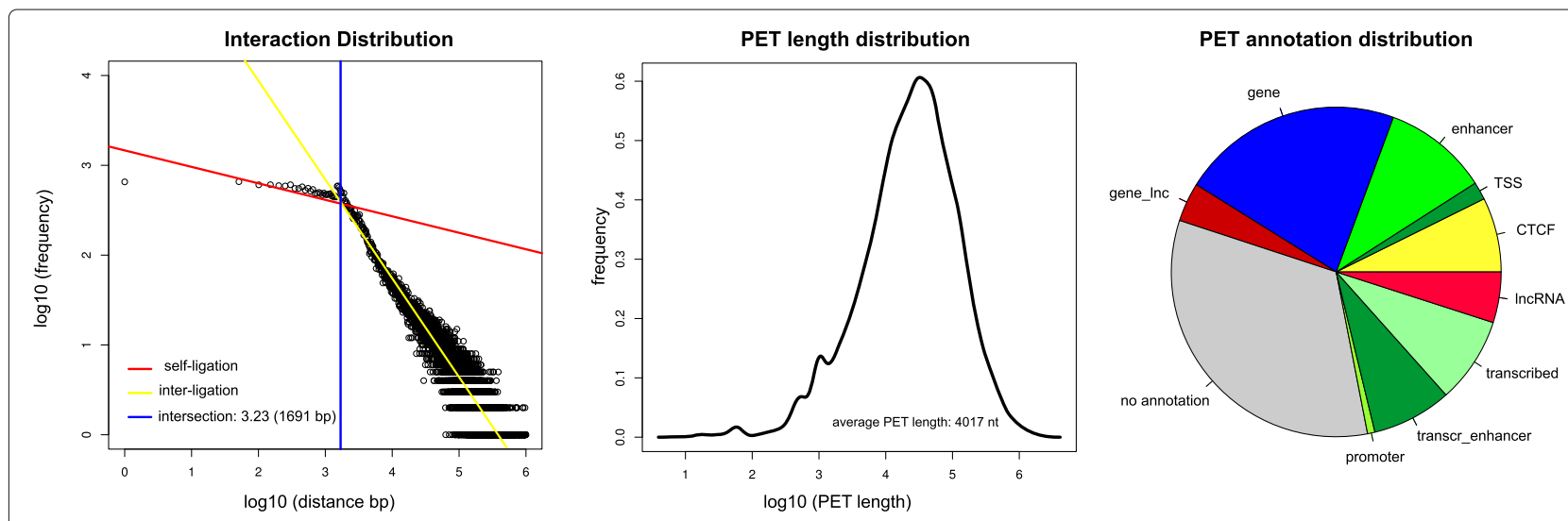

Fig. 2 Filtering of interacting regions. Left panel: Fitted mixture model to classify PETS in self-ligation and inter-ligation. Middle panel: Distribution of inter-ligation PET fragments' length. Right panel: Relative abundance of ChIA-PET fragments across different genomic annotations on the chromatin network 


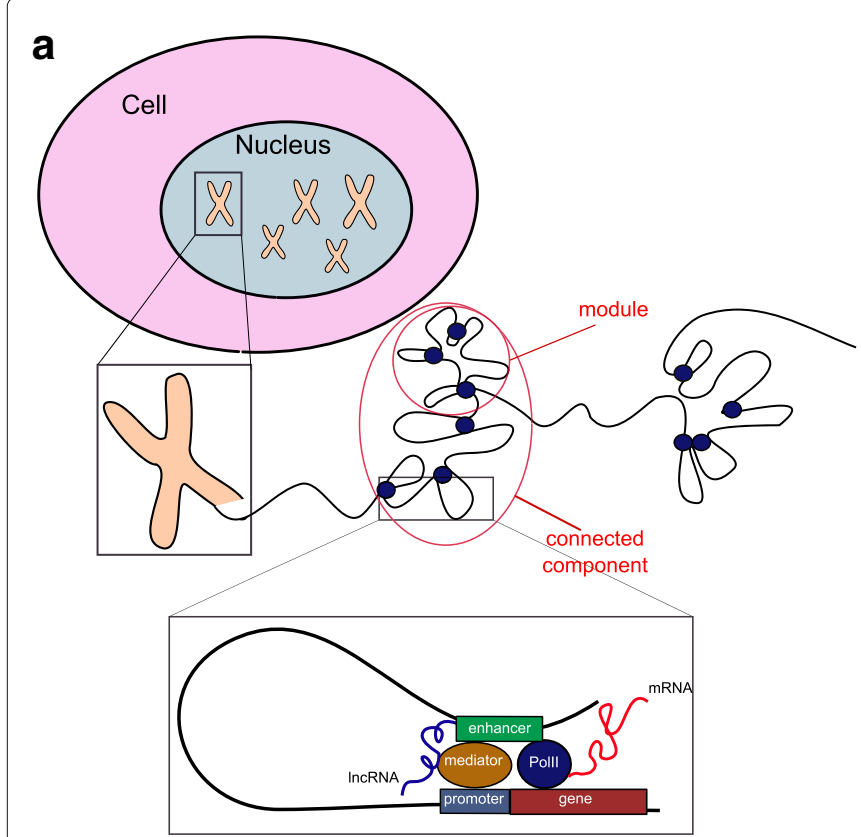

\section{b}

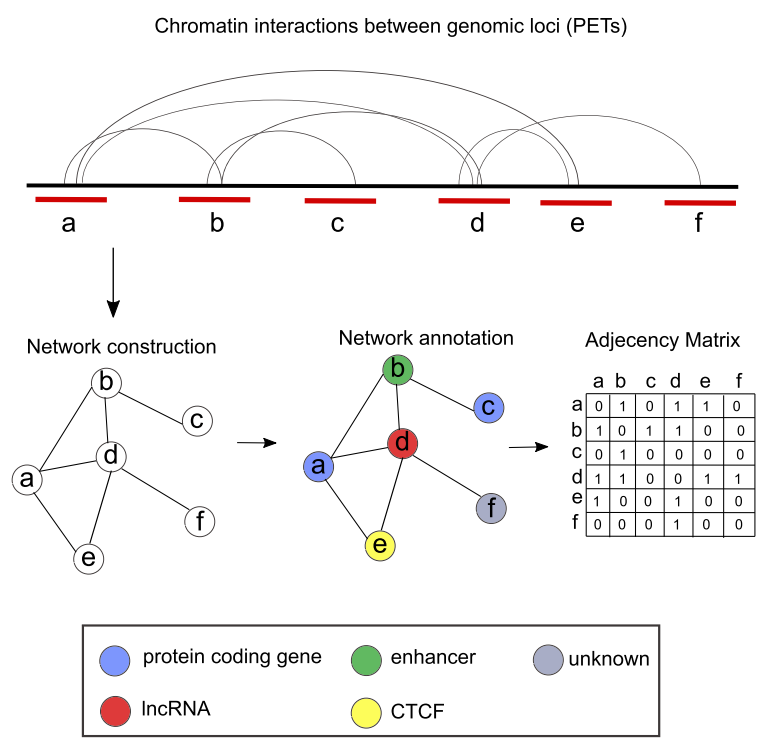

Fig. 3 Construction and annotation of the chromatin graph. a Modular organization of chromatin on each chromosome with highlight on looping between regulatory elements such as enhancers and promoters mediated by Polll, Mediator and nascent IncRNAs. b Steps involved in network construction and annotation from ChIA-PET data

lncRNADisease (as of June 2015) [30], where we used both experimentally validated associations between lncRNAs and diseases, as well as predicted associations. LncRNAs that were part of positionally conserved pairs of genes and lncRNAs were obtained from [31]. Additional annotations, such as functional lncRNAs in K562, VISTA and FANTOM5 enhancers, enhancers annotated from other sources [32], cancer risk Single Nucleotide Polymorphism (SNP) annotation and mouse orthologs we taken form Liu et al. [33].

\section{Network analysis of the chromatin graph}

Centrality measures For graph analysis we use standard graph concepts of interest for biological network analysis, see, e.g., [34] and [22]. To identify nodes of potential functional importance, we first look for nodes with a high degree, i.e., with a high number of incident edges, also called hubs. For each node $v$ in a graph $G=(V, E)$ we calculate the number $d(v)$ of edges incident to $v$ and call it its degree or degree centrality. For capturing the importance of a node $v \in V$ as an efficient connector between other nodes in the network we consider its betweenness centrality. It is defined as $b(v)=\sum_{s \neq v \neq t}\left(\sigma_{s t}(v) / \sigma_{s t}\right)$, where $\sigma_{s t}$ is the number of shortest paths from node $s$ to node $t$ and $\sigma_{s t}(v)$ is the number of such paths that pass through $v$.

MSM clustering for module detection Apart from single node characteristics, we are interested in sets of nodes forming functional units. A connected component $C=$
$\left(V_{C}, E_{C}\right)$ of a graph is defined as an inclusion-wise maximal subgraph of $G$ such that there exists a path between $v$ and $w$ for all vertices $v, w \in V_{C}$. If such a component is rather large, it often consists of so-called modules, i.e., subgraphs that have a high intra-connectivity but are only sparsely connected to the rest of the network. The modules are thus good candidates for functional units.

In this paper, we apply the MSM clustering method developed in [23, 24] on large connected components for finding modules. It is based on finding markov state models of a time-continuous random walk process. More precisely, it identifies modules as regions of the network where the process is metastable, i.e. trapped for a longer period of time. To this end, the number of network modules can be induced from the number of dominant eigenvalues of the generator matrix that governs the dynamics of the random walk process. Unlike most of the common approaches, MSM finds fuzzy instead of complete partitions of the network into modules, where some nodes are not uniquely assigned to exactly one of the modules, but can belong to several modules or to none. This allows to also capture intermodular nodes whose functional significance lies in mediating interactions between modules.

For every node $x$ we can calculate a value $q_{i}(x)$ as the random walk based probability of affiliation of a node $x$ to a module $M_{i}$. We then use a free parameter $\theta$ to refine the partitioning, i.e. we assign a node $x$ to a module $M_{i}$ 
if $q_{i}(x) \geq \theta$. If $\theta=1$ we obtain subgraphs exhibiting the strongest cohesiveness. By decreasing $\theta$ we expand modules until we reach a full partitioning of a graph by associating each vertex from the transition region with exactly one module it most likely belongs to. Fuzzy affiliation functions $q_{i}, i=1, \ldots, m$ can be obtained by solving sparse, symmetric and positive definite linear systems $([23,35])$.

Another free parameter is a resolution parameter $\alpha$, indicating how densely connected the modules we are interested in finding should be. For high values of $\alpha$ the method finds dominant, highly intraconnected modules and by decreasing $\alpha$ it finds also less pronounced modules. This is connected to the timescale at which the random walk leaves the transition region. It can be originally set according to the gap in the dominant spectrum of the generator of the random walk and then varied to observe the effect on the modules. In our application, it usually ranges from 100 to 2000.

Empirical Optimization criteria The parameters $\theta$ and $\alpha$ allow for an adaptation of the clustering to the specific application by integrating additional information on the networks nodes beyond the characteristics given by the network topology. Since we are looking for regulatory units involving lncRNAs, we chose to compare co-expression levels of intra- versus inter-modular genegene, lncRNA-gene and lncRNA-lncRNA pairs in order to find the best clustering parametrization. We argue that elements within the same module should have more correlated expression profiles, indicating co-regulation or potential mutual regulation, whereas intermodular node pairs are more independently regulated. In detail, we performed the MSM clustering for connected components from all chromosome graphs for a range of $\alpha$ and $\theta$ combinations. We chose the best combination by optimizing an empirical objective function (Eq.1) defined by the ratio of the median intra-module Mutual Information (MI) and the inter-module MI for all gene pairs in the connected component.

$$
\{\theta, \alpha\}_{\text {best }}=\operatorname{argmax}_{\theta, \alpha} \frac{\text { median }(\text { intra_MIs })}{\text { median }(\text { inter_MIs })}
$$

MI values between variables $X$, RPKM expression vector of gene1/lncRNA1 across 24 tissues and $Y$, RPKM expression vector of gene $2 / \operatorname{lncRNA} 2$ across 24 tissues, is defined in terms of their marginal Shannon entropies $H(X)$ and $H(Y)$ and their joint entropy $H(X, Y)$, as implemented in scikit-learn python package:

$$
M I(X, Y)=H(X)+H(Y)-H(X, Y)
$$

The entropy can explicitely be written as:

$$
H(X)=-\sum_{i=1}^{n} p\left(x_{i}\right) \ln p\left(x_{i}\right)
$$

where $x_{i}$ are the possible values of random variable $X$ with probability mass function $p(X)$. In detail, we apply a Gaussian smoothing to the histogram from the distributions of $X, Y$ and joint $(X, Y)$ and compute the entropy rather on the continuous distribution as described in [36].

LncRNAs tended to be more cell type-specific than protein-coding genes (Additional file 1: Figure S1a, b) and this might bias the MI computation (Additional file 1: Figure S1c). Computing the MI ratio on all gene pairs provides a more robust value. The reported ratio in Eq.1 for a connected component serves also as indicator for the quality of the clustering, where a high score implies a better partitioning with respect to MI and a ratio of at least one is expected for biologically meaningful clusterings. The best values for $\alpha$ and $\theta$ for each inspected connected component are reported in the table of Additional file 2, together with other properties of the detected clusters. We observe that generally clusterings with $\theta=$ 0.7 and small $\alpha$ (around 100-500), allowing more sparsely connected and relaxed modules, provide the highest MI ratio.

\section{Comparison with other clustering methods}

We compared our MSM clustering approach to other state-of-the-art clustering methods with respect to the mutual information ratio, which reflects our expectation that nodes connected in a module have correlated expression profiles. It is important to note again that our primary goal is to find modules that could represent functional units. To allow for and strengthen such an interpretation we consider co-expression of the involved nodes. The MSM approach allows us to integrate this aspect directly in the module detection by optimizing its parameters using MI ratios. This is a distinct advantage of our chosen method that is not directly reproducible by most commonly used clustering methods. We nevertheless need to consider whether other approaches might still yield more appropriate modules with respect to their co-expression in order to choose the most suitable method for our analysis.

We used the following methods and their implementation from the R igraph package [37]:

- cluster_fast_greedy function (FG), which finds dense subgraphs by directly optimizing a modularity score Q. Given a set of modules, $Q$ is computed as the ratio between the fraction of within-community edges versus the expected fraction of connections for the randomized network [38].

- clustering via Edge Betweenness (EB), cluster_edge_betweenness function, which is based on iteratively removing edges with highest edge betweenness from the graph [39], in order to hierarchically split the graph into modules. 
- leading eigenvalue clustering algorithm (EV), cluster_leading_eigen function, which implements the popular graph clustering method from Newman [40]. This method finds network modules by calculating the leading non-negative eigenvector of the so called modularity matrix.

- Walktrap algorithm which is a Repeated Random Walk (RRW) based clustering, cluster_walktrap function. Similarly to our MSM algorithm this approach finds modules in a graph by exploiting metastability of the random walk [41], but uses only a time-discrete version of the process.

We compare these methods to our MSM procedure using the largest connected component of our chromatin graph on chromosome 1. As mentioned this comparison is not straightforward since, firstly, none of these methods support fuzzy clustering as in the MSM approach. In particular, the modularity score $Q$ which most of these methods use is hard to compare between fuzzy and non-fuzzy clustering and might not be very meaningful in our context. Secondly, the other approaches do not allow us to optimize for MI ratio in an integrated fashion that would impact size and number of modules.

To address these issues, we evaluated a range of different modules for each of the considered methods from the igraph package, mimicking optimization for MI ratio. First, we run each algorithm unbiased and assess the modules returned by the optimization algorithm underlying the method. As additional information to this clustering, most of the considered algorithms return a hierarchical overview of the best clusterings for a range of different module numbers - comparable with the variation of the parameters of MSM. This allows us to assess the results for clusterings corresponding to a range of module numbers from 8 to 24 in incremental steps of 4. An exception to this procedure is the EV algorithm that does not offer a simple way to change the number of modules. Rather, we can only influence this number indirectly using the 'steps' parameter, which can only increase the number of modules until an upper limit is reached. The resulting MI ratios are visualized in Fig. 4. In a second type of assessment, we transfered the information on module number we derived from our MSM approach after optimizing for MI ratio to the other approaches, meaning, we enforced the module number we found with MSM for the other approaches. The outcome of this assessment can also be seen in Fig. 4 marked in red.

Our mehtod returns on average the highest MI ratio compared to other methods (Fig. 4). It is noteworthy that the clustering with the number of modules reported by MSM is often the best clustering and always better or equal to the default clustering.

\section{Module functional enrichment analysis}

GO functional enrichment and pathway analysis from the KEGG database for the genes contained inside each identified module was done with the $\mathrm{R}$ package GSEABase [42], in order to transfer functional annotation gained from the genes to the IncRNAs contained in the same module. Only enriched terms with adjusted $p$-values lower or equal than 0.1 and having more than two genes from the module annotated with that term are reported in Additional file 2. Nodes not uniquely assigned to a single cluster, but belonging to the transition region defined above, can be also functionally annotated by transferring annotation from their direct neighboring genes.

\section{Results}

In this section we first focus on the analysis of different centrality measures for lncRNA nodes and other annotations, as well as "connectors" lncRNAs of high betweenness. We show that network properties are related to specific regulatory annotations as well as biological functions. Next, we exploit the modularity of the K562 ChIA-PET interaction network to identify network modules including potentially functional lncRNA with fuzzy MSM clustering applied to each chromosome's biggest component, while still taking into account gene co-expression. Finally, in the absence of an high-throughput gold standard of validated lncRNA functions, we discuss some lncRNA-gene target interactions retrieved manually from the literature and contained in our detected modules, as well as the potential functional importance of inter-modular nodes, which is a unique feature of our approach. We also provide some general means on how to mine the network and the modules to gain a better clue into unknown lncRNA functions.

\section{Hierarchical graph analysis of the ChIA-PET interaction network}

When plotting the frequency of interactions at different genomic distances (Fig. 2, Left panel) one can clearly distinguish two linear 'regimes', corresponding to a mixture distribution of PETs where two different linear functions can be fitted. The intersection of the two fitted lines in the log-log plot was chosen as cutoff to differentiate self-ligation, corresponding to short range ChIA-PET interactions, from inter-ligation, corresponding to long range interactions. Self-ligation PETs were excluded from the network analysis as, in most of the cases, they do not correspond to chromatin interactions between different genomic segments. Most of the remaining PETs could be annotated as either genes or lncRNAs or other regulatory elements, while about one third of them could not be assigned to any genomic or regulatory annotation (Fig. 2 right panel). In total, 6500 lncRNAs were expressed above the threshold (see "Methods") in K562 cells, but only 


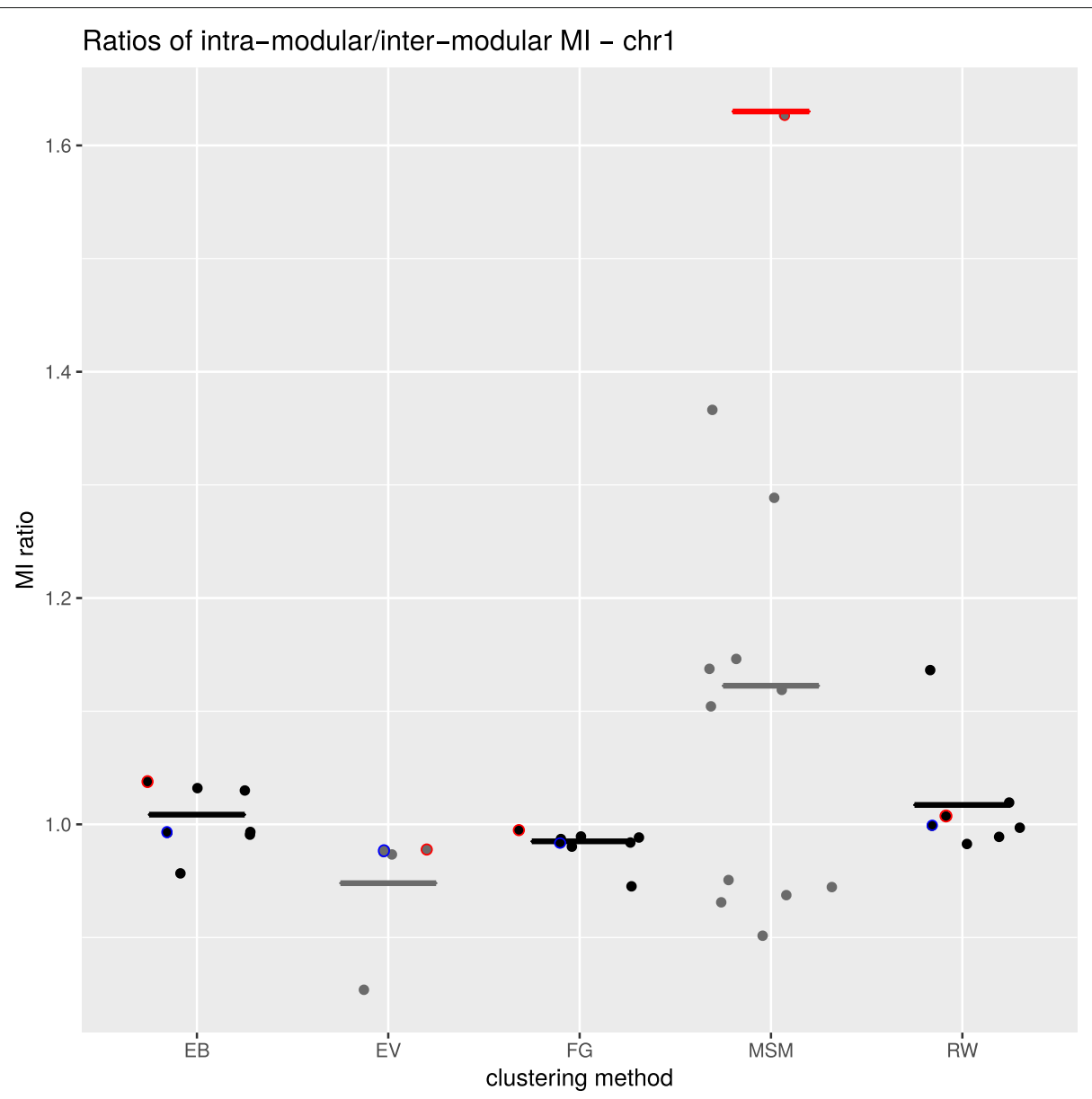

Fig. 4 Comparison of different graph clustering methods. Our MSM clustering approach is compared to other methods from the igraph package (EB - clustering via edge betweenness; EV-eigenvalue clustering; FG-fast and greedy clustering; RW-random walk clustering). All methods are run with different ranges of parameters and/or number of modules, and the mutual information (MI) ratio is computed for every scenario as described in Material and Methods. For each method the distribution of the resulting Ml ratio is shown, together with the median value (horizontal line). For each clustering method the result obtained with the MSM's optimal number of modules is circled in red and the results obtained with its own optimization is circled in blue. The red line indicates the best partition for our MSM clustering, i.e. values of $\alpha$ and $\theta$ yielding the highest MI ratio

3229 were found to be involved in ChIA-PET interactions. About $40 \%$ of the lncRNA-nodes could be annotated with more than one lncRNA (mainly one of the sense and the other on the reverse strand).

To cope with the size and heterogeneous nature of the chromatin graph we developed an hierarchical analysis approach that enabled us to add step-wise resolution to subgraphs of interest guided by the results of the previous step (Fig. 1). First, we analyzed the chromatin graph (Table 1) to identify global hubs by computing the degree centrality of lncRNAs and other genomic elements. An overview of the general properties of the chromatin graph is given in Table 1 . The chromatin network is very sparse, with many components representing singleton nodes or containing very few nodes. When looking at the chromatin graph, we notice that only few lncRNAs have a degree centrality higher than 10 , while the majority of lncRNAs exhibits a degree between one and three (Additional file 1: Figure S1d). The logarithmic visualization of degrees in Additional file 1: Figure S2 middle panel matches the general observation that in biological networks degrees are often distributed according to a power law, i.e., there exist few hubs and many much less densely connected nodes [22]. A comparison of degree distributions for lncRNAs, protein coding genes, enhancers, promoters/transcribed regions and CTCF sites (Additional file 1: Figure S2) showed that protein-coding genes had the largest degree, constituting the main network's hubs, followed by lncRNAs (both gene-overlapping and intergenic ones), enhancers, promoters and lastly CTCF sites. Nodes with different annotations followed a power law with similar exponents, except nodes annotated with CTCF sites, probably to reflect the different biological role of such binding sites, as chromatin barriers or insulators [43] with 
Table 1 Properties of the chromatin graph

\begin{tabular}{|c|c|c|c|c|c|c|c|c|}
\hline chr & no.cc & $\begin{array}{l}\text { Min } \\
\text { cc } \\
\text { csize }\end{array}$ & $\begin{array}{l}\text { Mean } \\
\text { cc } \\
\text { csize }\end{array}$ & $\begin{array}{l}\text { Max } \\
\text { cc } \\
\text { csize }\end{array}$ & $\begin{array}{l}\text { Number of } \\
\text { nodes } \\
\text { containing } \\
\text { IncRNA }\end{array}$ & $\begin{array}{l}\text { Nodes } \\
\text { containing } \\
\text { IncRNA involved } \\
\text { in interactions }\end{array}$ & $\begin{array}{l}\text { Node containing } \\
\text { IncRNA with } \\
\text { highest degree }\end{array}$ & Degree \\
\hline chr1 & 1765 & 1 & 3.35 & 503 & 444 & 404 & $\begin{array}{l}\text { RP11- } \\
\text { 442N24_B.1,RNU11 }\end{array}$ & 26 \\
\hline chr2 & 1264 & 1 & 2.77 & 64 & 299 & 250 & ZFP36L2 & 19 \\
\hline chr3 & 994 & 1 & 2.82 & 70 & 213 & 185 & TERC & 13 \\
\hline chr4 & 649 & 1 & 2.66 & 52 & 160 & 127 & $\begin{array}{l}\text { RP11- } \\
\text { 539L10.3,AC093323.3 }\end{array}$ & 9 \\
\hline chr5 & 890 & 1 & 2.71 & 43 & 245 & 197 & ARRDC3 & 15 \\
\hline chr6 & 1172 & 1 & 3.21 & 337 & 208 & 190 & ABT1 & 14 \\
\hline chr7 & 1016 & 1 & 2.76 & 88 & 196 & 164 & LINC01287 & 14 \\
\hline chr8 & 619 & 1 & 2.87 & 105 & 187 & 159 & YWHAZ & 20 \\
\hline chr9 & 505 & 1 & 3.1 & 141 & 127 & 118 & SNHG7 & 11 \\
\hline chr10 & 685 & 1 & 2.84 & 92 & 144 & 126 & VIM & 9 \\
\hline chr11 & 858 & 1 & 3.03 & 128 & 216 & 188 & BEST1,FTH1 & 12 \\
\hline chr12 & 655 & 1 & 3.25 & 69 & 224 & 199 & BTG1 & 22 \\
\hline chr13 & 280 & 1 & 2.45 & 39 & 62 & 51 & MIR17HG & 21 \\
\hline chr14 & 357 & 1 & 2.98 & 36 & 131 & 115 & PRMT5 & 10 \\
\hline chr15 & 430 & 1 & 3 & 72 & 156 & 132 & GABPB1 & 9 \\
\hline chr16 & 457 & 1 & 3.63 & 196 & 182 & 166 & RAB26,TRAF7 & 17 \\
\hline chr17 & 517 & 1 & 4.3 & 350 & 275 & 267 & LINC00910 & 17 \\
\hline chr18 & 278 & 1 & 2.61 & 71 & 61 & 47 & MYL12A & 17 \\
\hline chr19 & 380 & 1 & 5.36 & 158 & 227 & 220 & SLC1A5 & 15 \\
\hline chr20 & 407 & 1 & 3.18 & 90 & 109 & 99 & CEBPB & 26 \\
\hline chr21 & 207 & 1 & 3.02 & 53 & 73 & 62 & DYRK1A & 7 \\
\hline chr22 & 279 & 1 & 3.24 & 67 & 84 & 78 & POLDIP3 & 20 \\
\hline $\operatorname{chr} x$ & 358 & 1 & 2.75 & 60 & 57 & 49 & VSIG4 & 10 \\
\hline
\end{tabular}

For each chromosome we report: the total number of connected components (no.cc), the minimum number of nodes (min cc csize), the average number of nodes (mean cc csize) and maximum number of nodes (max cc csize)) of the connected components, the total number of annotated IncRNAs (number of IncRNAs), the total number of IncRNAs which are involved in at least one interaction (IncRNAs in interactions), the IncRNA gene symbol of the highest degree's IncRNAs (IncRNA with highest degree) and the actual highest degree value for that IncRNA (degree)

respect to other genomic annotations. For future studies, the top 20 highest-degree lncRNAs from the chromatin network are listed in Table 2.

Since the chromatin graph decomposes in a natural way into the graphs representing the single chromosomes, we compute the lncRNA degree chromosome-wise. Even nodes that are not among those of highest degree in the chromatin graph may be distinguished with respect to their chromosome graph. Second, we focus on the connected components containing lncRNAs of each chromosome graph to obtain the next resolution level. Small components are then amenable to a full analysis of different aspects of interest, while for large connected components we still need indicators that guide our search for important lncRNA modules. In (Additional file 1: Tables S2, S3 and S4) we report this analysis for the biggest connected components of chromosome 1, 17 and 11, respectively. In addition, we evaluate the betweenness centrality of each lncRNA node. Among lncRNAs with high betweenness in their respective connected component we find MALAT1, SHG16, RNU11 and RP11-400F19.8, known oncogenes, as well as lncRNAs of unknown function, such as LINC00910, RP11-442N24 and RP4-798A10.7. Interestingly, PETs annotated as IncRNAs, which overlapped also a protein coding gene, either on the same or the anti-sense strand, had on average the highest betweeness compared to other genomic classes, including protein coding genes (Additional file 1: Figure S2 right panel, Table S1). This points to the important central role of these regions with dual genomic annotation (coding/noncoding) as linkers and communicators between different regulatory modules in the ChIA-PET network. Finally, 
Table 2 Top 20 IncRNAs with highest degree from the chromatin graph

\begin{tabular}{|c|c|c|c|c|c|c|c|}
\hline IncRNA name & Degree & $\begin{array}{l}\text { To-gene } \\
\text { degree }\end{array}$ & Chormosome & Annotation & RPKM & Conserved & Disease \\
\hline RP11-442N24_B.1,RNU11 & 26 & 9 & chr1 & Inc_transcr_enhancer & 227.4193 & no & yes \\
\hline RP4-798A10.7 & 21 & 5 & chr1 & Inc_transcr_enhancer & 16.0232 & no & no \\
\hline MIR17HG & 21 & 1 & chr13 & Inc_transcr_enhancer & 518.9101 & no & yes \\
\hline LINC00910 & 17 & 5 & chr17 & Inc_transcr_enhancer & 37.0585 & no & no \\
\hline RP11-1082L8.4 & 16 & 2 & chr8 & Inc_transcr_enhancer & 1.2984 & no & no \\
\hline LINC01287 & 14 & 0 & chr7 & Inc_transcr_enhancer & 77.8624 & no & no \\
\hline TERC & 13 & 3 & chr3 & Inc_CTCF & 111.0789 & no & yes \\
\hline AC073283.4 & 13 & 3 & chr2 & Inc_transcr_enhancer & 0.156 & no & no \\
\hline RP11-495P10.3 & 12 & 0 & chr1 & Inc_promoterFI & 1.0816 & no & no \\
\hline RP11-301G19.1 & 12 & 0 & chr6 & Inc_transcr_enhancer & 914.2072 & no & no \\
\hline KB-1732A1.1 & 12 & 1 & chr8 & Inc_transcr_enhancer & 1.0105 & no & no \\
\hline SNHG7 & 11 & 5 & chr9 & Inc_TSS & 112.0253 & no & no \\
\hline BZRAP1-AS1 & 11 & 0 & chr17 & Inc_transcr_enhancer & 6.9533 & no & no \\
\hline RP11-247A12.2 & 10 & 3 & chr9 & Inc_transcr_enhancer & 0.3745 & no & no \\
\hline CTD-2587H24.5 & 10 & 8 & chr19 & Inc_transcr_enhancer & 0.1669 & no & no \\
\hline CTD-2587H24.10 & 10 & 7 & chr19 & Inc_transcr_enhancer & 17.1446 & no & no \\
\hline SNHG16,RP11-666A8.8 & 9 & 4 & chr17 & Inc_promoterFI & 73.5648 & no & yes \\
\hline SNHG12 & 9 & 7 & chr1 & Inc_TSS & 229.3524 & no & no \\
\hline RP5-884M6.1 & 9 & 2 & chr7 & Inc_transcr_enhancer & 30.2113 & no & no \\
\hline RP11-539L10.3,AC093323.3 & 9 & 6 & chr4 & Inc_transcr_enhancer & 92.453 & no & no \\
\hline
\end{tabular}

For each IncRNA we report its degree centrality (degree), its degree centrality computed only from gene connections (to-gene degree), the chromosome it belongs to (chromosome), its annotation based on chromatin segmentation (annotation), its expression value (RPKM) in the K562 cell line (expression), whether it is positionally conserved according to $\mathrm{X}$ et al. [31] (conserved), and whether it is known from databases or literature its involvement in diseases(disease)

to identify relevant functional units we conduct a module search using the MSM clustering method described above.

\section{Network analysis and biological properties of IncRNAs}

By manually inspecting the functional annotation of the top 20 expressed lncRNAs with highest degree, we find several lncRNAs known from previous studies to be cancer-associated. For example, RNAs from the SNHG family important in cell proliferation and invasion in different cancer types [44]; RP11-301G19.1, over-expressed in leukemia [45]; TERC, involved in telomerase activity and associated to leukemic cells [46], and the intergenic lncRNA MIR17HG, host transcript of the MIR17-92a-1 cluster, known to be involved in cell survival and cancer proliferation [47]. However, disease annotation is sparse and limited for lncRNAs compared to protein-coding genes. The fraction of intergenic long noncoding RNAs (lincRNAs) from the ChIA-PET network, that could be annotated with a disease in our analysis (see "Methods" section for more details) was only 9\% (217 out of 2305), therefore it is hard to systematically access whether high-degree lncRNAs are significantly associated to diseases. Comparing the degree distribution of lincRNAs annotated with a disease versus lincRNAs not linked to a disease we do not observe any significant associations ( $p$-value $=0.384$, Wilcoxon rank sum test). When we perform the same analysis including also lncRNAs overlapping protein-coding genes, we can assign a disease up to $42 \%$ of the lncRNAs in our network, and obtain a significant association between degree centrality and disease annotation ( $p$-value $<1.22 * 10^{-16}$, Wilcoxon rank sum test, Additional file 1: Figure S3).

A recent study from Liu et al. [33] investigates the functional importance of lncRNAs, mainly as trans regulators of gene expression, by performing CRISPR interference and targeting thousands of lncRNA loci in seven diverse cell lines, including K562. We partly used these data to explore other biological properties of our ChIA-PET network. Liu et al. define functional lncRNAs or 'hits' those which showed a significant phenotype, i.e. affecting cell growth, in a cell-type specific manner. K562 hits were enriched in the chromatin graph, compared to non-hits (odd ratio $=2.07, \mathrm{p}=0.008$, Fisher's exact test), but did not have significantly higher degree centrality. K562 lncRNAs annotated by Liu et al. to be in close genomic proximity to cancer risk SNPs were also enriched in the chromatin network compared to lncRNAs far from those SNPs (odd 
ratio $=2.65, \mathrm{p}=1.2 * 10-5$, Fisher's exact test) but did not have significantly higher degree.

LncRNAs annotated as enhancers from chromHMM were enriched for tissue-specific expression (odd ratio $=2.4, p$-value $=1.4^{*} 10-7$, Fisher's exact test) and had significantly higher degree centrality compared to lncRNAs which did not overlap enhancer elements ( $p$-value $=5.4 * 10^{-62}$, Wilcoxon rank sum test, Additional file 1: Figure S3). This still holds when considering also lncRNAs overlapping protein coding genes $\left(p\right.$-value $=1.3 * 10^{-48}$, Wilcoxon rank sum test, Additional file 1: Figure S3). The significant associations between high degree and enhancer annotation of lncRNAs in the network holded also for FANTOM5 enhancers $(p$-value $=0.034$, Wilcoxon rank sum test $)$ and enhancers defined by other studies ( $p$-value $=2.1 * 10^{-9}$, Wilcoxon rank sum test), as well as super-enhancers ( $p$-value $=4.5 * 10^{-10}$, Wilcoxon rank sum test) [32]. This suggests that enhancer-like lncRNAs are hubs in the Pol II-mediated ChIA-PET network and connect several regulatory regions to gene loci in an extensive and combinatorial fashion. In addition, IncRNAs which overlapped both 'enhancer' and 'transcribed' annotations from chromHMM, which we denote as 'transcribed enhancers', had a significantly higher degree compared to those lncRNAs annotated only as 'enhancer' but not 'transcribed' from chromHMM ( $p$-value $=1.9 * 10^{-19}$, Wilcoxon rank sum test). All these findings are in line with the results from Liu et al., where the authors show that enhancer proximity and chromosomal contacts correlate with lncRNA function [33], and that the transcription of the lncRNA itself might confer, in some cases, the regulatory potential to the lncRNA genomic locus.

Positionally conserved lncRNAs from our network, defined as lncRNAs located close to genes which are orthologous between human and mouse [31], had also a significantly higher degree compared to the non positionally conserved ones ( $p$-value $=0.044$, Wilcoxon rank sum test, Additional file 1: Figure S3). Finally, also lncRNAs which had a direct orhtologous gene in mouse [33] had significantly higher degree than the rest ( $p$-value $=0.037$, Wilcoxon rank sum test), highlighting the importance of evolutionary conserved lncRNAs in the ChIA-PET chromatin network. Positionally-conserved IncRNAs have also been associated with developmental or cancer genes, and shown to be in chromatin loops, which contact enhancer-regulatory sequence. In our network, we observe that IncRNAs which are also annotated as positionally conserved, have a significantly higher degree than not positionally conserved ones ( $p$-value $=0.044$, Wilcoxon rank sum test $)$, indicating their potential role as functional hubs in the Pol II chromatin network.

\section{Small network components contain validated IncRNA-gene functional interactions}

Prior to cluster analysis, we first inspected small connected components of the network (sub-graphs of the order of tens of nodes) to assess whether the spatial proximity in the Pol II ChIA-PET network recapitulates some well known lncRNA-gene target interactions from the literature. The lncRNA transcript overlapping the longest intron of the AHI1 gene has been shown to significantly impact the expression of the BCLAF1 in K562 from a CRISPRi experiment [33]. Here we show that this regulation is mediated by a direct ChIA-PET interaction in a small connected network module on chromosome 6 (Additional file 1: Figure S4a)). In addition, other ChIA-PET modules contain validated interactions of lncRNAs with their target genes, for example PVT1 with its known target MYC [33] on a small connected component on chromosome 8 (Additional file 1: Figure S4b)). A small module on chromosome 1 contains the lncRNA CYP4A22-AS1 (Additional file 1: Figure S4d)), also known as ncRNA-a3, which has been shown to act as enhancer for its flanking stem cell leukemia-associated gene TAL1 [13], and we recapitulate the direct interaction between them. The active enhancer-like lncRNA linc00853, also known as ncRNA-a4 is also part of the ncRNA-a3 network (Additional file 1: Figure S4d)) and directly regulates its flanking gene CMPK1, as already previously verified experimentally [13], suggesting a synergistic action of these two lncRNAs in coordinating the transcriptional activity of a group of four genes in this module.

Finally, we looked at the well characterized lncRNA Xist (Additional file 1: Figure S4e)), known to be involved in transcriptional gene silencing during X-chromosome inactivation. From our analysis it is evident, given the lack of ChIA-PET interactions, that Xist does not associate to Pol II to regulate its target genes in an enhancer-like fashion, as expected given its suggested silencing function. On the other hand, we could recover direct Pol II-mediated interactions between XIST and lncRNA FTX, JPX and TSIX (Additional file 1: Figure S4e) which are known regulators of XIST transcription [11].

\section{Analysis of IncRNA-containing modules}

For the biggest components of each chromosome we performed MSM clustering as described in Material and Methods and inspected the resulting modules for functional annotation. One way to gain functional clues about uncharacterized lncRNAs is to inspect the functions of its interacting genes or the genes contained in the same module via GO/KEGG term enrichment. Many of the identified clusters in our network were enriched in cancer-related terms (Additional file 2). The most abundant KEGG pathway from our analysis "Chronic myeloid leukemia" is found in ten modules, in line with K562 
being a leukemia cell line, though only two modules in chromosome 17 contained more than one gene in the pathway. The more general term "Pathways in cancer" was enriched in four modules (see Additional file 2, sheet 2). We found more enriched terms linked to cancer, such as "mTOR signaling pathway", "Jak-STAT signaling pathway", "hematopoietic stem cell differentiation" and "response to interleukin-15" (hematopoietic growth factor (Additional file 2).

\section{Examples of modular structures and putative IncRNA functions on chromosomes 20, 1, 17 and 11}

We detect two modules on the biggest component of chromosome 20, where cluster 1 contains the validated functional interaction between TRERNA1 IncRNA and the SNA1 gene [8], mediated by several enhancer elements (Additional file 1: Figure S4c). We detect 11 modules for the biggest component on chromosome 1 obtained with setting $\alpha=1000$ and $\theta=0.8$ (Fig. 5b). Different clusters are recognizable by different colors of their intra-cluster edges. Cluster 6 in Fig. $5 \mathrm{~b}$ is the regulatory module containing the well-known eRNA FALEC, which has been shown to harbor enhancer-like functions and significantly influence the expression of its flanking gene ECM1 [13], also present in the same module. By inspecting the interactions in this module, we learn that the interaction between FALEC and the ECM1 locus is mediated by the ADAMTSL gene. Interestingly, the Myeloid Cell Leukemia apoptosis regulator MCL1 and the Hypoxia-Inducible factor 1-Beta ARNT are also in the same module, indirectly linked to FALEC via other lncRNAs, protein-coding genes and several transcribed enhancer elements (colored in green in Fig. 5b). The module containing FALEC is enriched in functional terms related to chromatin silencing and negative regulation of gene expression, highlighting a role of this lncRNA in epigenetic-related processes. Other interesting modular units on chromosome 1 comprise: cluster 3, containing the lncRNA of unknown function FLJ37453 which is connected via intra-modular interactions to SPEN, known to associate to lncRNAs (e.g. Xist) and mediate gene repression [48]; cluster 2, containing, among others, lncRNAs SNHG12, ubiquitously expressed in several cancers and two other lncRNAs of high degree, RUN11 and RP11$442 \mathrm{~N} 24$, as well as genes interestingly enriched in functions associated with lipid metabolism, transcription termination and p53-mediated signal transduction; cluster 10 , containing the high degree lncRNA RP4-798A10.7 and genes with enriched functions related to chromatin and nucleosome assembly, suggesting a role of this lncRNA in shaping chromatin organization.

Clustering of the biggest component on chromosome 11 (Fig. 5d) resulted in an optimal partition of four dominant modules, obtained with setting $\alpha=1000$ and $\theta=0.7$ (thus identifying more relaxed modules). Particularly interesting are the modules in Fig. 5d) marked by red and light blue connections, namely cluster 1 and 3, linked by the oncogene lncRNA MALAT1, known to act as transcriptional regulator for numerous genes involved in cancer metastasis and cell migration [9]. MALAT1 has a degree of 8 , but exhibits a very high betweenness. This indicates that MALAT1 is important in the context of the entire connected components, not only for its first-order neighbors, but also because it brings different gene clusters in close proximity at the chromatin level. This fits well with the known role of MALAT1 as global regulator of cancer genes and orchestrator of a global transcriptional response [9]. While the physical interaction of lncRNA MALAT1 with the SIPA1 leukemia oncogene has been experimentally validated [49] and recapitulated in our network, MALAT1 is linked to other crucial oncogenes in the two linked modules, and co-expressed with NEAT1, another well-known lncRNA in the context of cancer [9].

Finally, we briefly discuss the clustering results of the biggest connected component of chromosome 17, obtained with $\alpha=700$ and $\theta=0.7$ (Fig. 5c), resulting in eleven modules. This component is particularly interesting because it contains several lncRNAs with very high degree/betweenness, so potential core players in the leukemia regulatory network, but of unknown function, such as LINC00854 and LINC00910. LINC00910 was already pointed in other studies as a highly connected IncRNA [50], observed to be linked to an upstream superenhancer [50,51] and hypothesized to be involved in immune related functions and lymphocyte activation [50]. In our network, it exhibits the highest degree in chromosome 17 and very high betweenness, it interacts with several transcribed enhancers and with many CTCF binding sites. It is also found in direct or indirect interactions with numerous known cancer genes, such as NBR1, BRACA1, ICT1, SUMO, NUP85 and others. As the genes contained in this detected module are enriched in the 'calcium signaling pathway' annotation, which is a key regulator of B lymphocyte fate in Leukemia [52] we propose a potential function for LINC00910 in the Leukemia's ChIA-PET as regulator of calcium signaling-related genes. However, experimental approaches so far could not identify genes which are significantly regulated by LINC00910 in the K562 cell line, neither in cis or in trans [33], and further experimental tools are needed to validate this hypothesis.

Of great interest in this component is IncRNA RP11400F19.8, a node with high degree and high betweenness, which was not assigned to any cluster by our method but belonged to the previously defined 'transition' region (Additional file 1: Table S5). Although not assigned to any module, RP11-400F19.8 is far from being a nonfunctional lncRNA, and was already identified in a previous transcriptome-wide association study as a cancer risk 


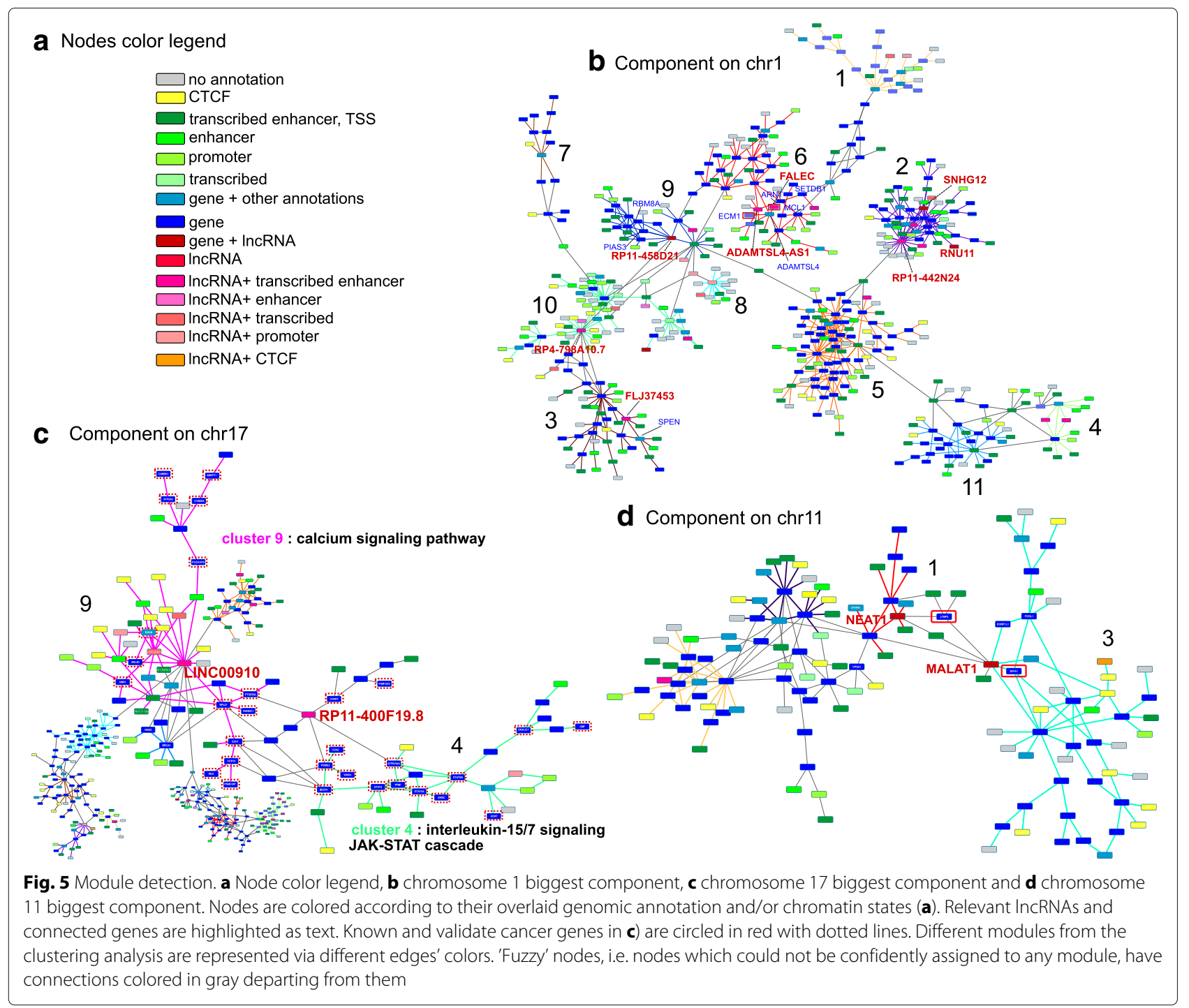

locus [53]. In our network RP11-400F19 links two modules enriched in different immune biological processes (Fig. 5c) and brings in close spatial proximity known cancer genes (denoted with dotted red circles in Fig. 5c)) from both modules.

\section{Discussion}

LncRNAs play key regulatory roles in a wide range of processes, and a small number of them has been shown to operate in the nucleus and influence transcriptional regulation of neighboring or distal genes. To which extent celltype specific 3D chromatin organization and other DNA regulatory elements contribute to lncRNA-mediated gene regulation has been poorly investigated. In addition, functional annotation for most of the annotated lncRNAs, as well as their role in gene regulatory networks remains elusive. Based on the fact that transcripts sharing common expression patterns should largely share similar biological pathways, a number of different studies have used the 'guilt by association' approach to functionally annotate lncRNAs based on expression similarities with proteincoding genes of known function.

Here we comprehensively map ChIA-PET chromatin contacts mediated by Pol II in the K562 cell line to lncRNAs, genes and other DNA regulatory elements, and propose a multi-step approach to analyze lncRNA regulatory functions using graph analysis techniques. We first show a significant association between some network properties of lncRNA-annotated nodes, such as degree or betweenness, and biological properties, such as enhancer functions of lncRNAs from multiple datasets. Afterwards, we inspect functional mechanisms of network modules. The added value of our approach is twofold. First, lncRNA-mediated transcriptional regulatory modules are identified by means of fuzzy clustering analysis directly on the chromatin network, providing a first link 
between transcriptional regulation and lncRNA association/functions at chromatin level. Second, although alternative choices exist for the module search, we decided on the MSM clustering since it does not impose an often artificial full partition of the network into modules, but outputs a fuzzy clustering which allows more flexible interpretation of lncRNA regulation. Also, the $\theta$ and $\alpha$ parameters of our clustering method are chosen to maximize gene co-expression inside the same module, allowing to incorporate additional information in the module refinement, beyond network topology. Our approach can identify direct IncRNA targets, as well as the regulatory modules they belong to, as shown here for known and novel lncRNAs. Although it is not often possible to discern modules of co-regulated genes/lncRNAs from causal lncRNA-mediated cis-regulation without experimental validation, and many lncRNAs identified in the modules might actually function in trans to regulate gene expression, our approach is promising in detecting cis-regulatory modules. In fact, a previous study investigating functional interactions of MALAT1 and NEAT1 with target genes via Capture Hybridization Analysis of RNA targets (CHART) sequencing in the MCF7 cell line shows that half of the interacting genes are located up to three hops of connectivity with their lncRNAs and in the same inter-connected chromatin cluster [50]. Unfortunately the same CHART data are not available for K562, but these observations, together with the evidence of validated direct and indirect lncRNA-gene target functional interactions in the clusters of our network, highlights that the detected modules are a good starting point to look for potential lncRNA-mediated cis-regulatory interactions. Our approach, presented here for the Pol II ChIA-PET network in K562, is generally straightforward enough to be applied to other factors, such as CTCF and other cell lines, and module analysis repeated for other connected components.

Incorporation in the future of other annotations in the network, such as Transcription Factor Binding Sites, will enable a better interpretation of individual modules.

Although the modular lncRNA regulatory code remains to be tested, investigating the connections between lncRNAs, genes and other regulatory elements are important steps towards further definition of lncRNA functions on a system-wide level. The investigation of modules related to lncRNAs whose functionality is not yet known can suggest new targets and the regulatory components involved in regulation. Therefore, we propose that our functional annotation scheme can be applied to thousands of lncRNAs in a tissue-specifc manner.

\section{Conclusion}

In this study we demonstrate that the integration of 3D chromatin interaction and co-expression analysis pro- vides a powerful network analysis approach for in silico functional analysis of both known and novel lncRNAs involved in transcriptional regulation. The results presented here, in particular the detected regulatory modules on the ChIA-PET interaction network, are an important resource for further biological research.

\section{Additional files}

Additional file 1: This pdf includes supplementary tables and figures referred to in the main text. This includes four additional figures, included one showing several small connected components pointing to IncRNA whose regulated genes are known from literature, as well as tables with network properties of IncRNAs in the biggest connected components of chromosomes 1, 11 and 17. (PDF $421 \mathrm{~kb}$ )

Additional file 2: In this excel file we provide the results from clustering analysis of the biggest connected component of each chromosome, in order to assist future experimental studies. For each component, we report the results from those values of $\alpha$ and $\theta$ yielding the best partition according to the $\mathrm{Ml}$ ratio criteria. We report the clustering parameters, the resulting $\mathrm{Ml}$ ratio, the number of obtained modules per component, the number of IncRNAs, protein-coding gene, as well as the overall number of nodes for each component. Note when the genomic coordinates of a gene and a IncRNA overlap, both the gene and the IncRNA name are reported for the same node. Sheet 2 of this file contains the results of $\mathrm{GO} / \mathrm{KEGG}$ enrichment analysis for the modules of each chromosome's biggest component. (XLSX $25 \mathrm{~kb}$ )

\section{Abbreviations}

CHART: Capture hybridization analysis of RNA targets; ChIA-PET: Chromatin interaction analysis by paired-end tag sequencing; CSHL: Cold spring harbour lab; EB: Edge betweeness; EV: Eigenvalue; FG: Fast greedy; GO: Gene ontology; KEGG: Kyoto enciclopedia of genes and genomes; lincRNAs: Intergenic long non-coding RNAs; IncRNAs: Long non-coding RNAs; Ml: Mutual information; MSM: Markov state model; ncRNAs: Non-coding RNAs; PETs: Paired-End tags; Pol II: Polymerase II; RBPs: RNA binding proteins; RNA-seq: RNA sequencing; RPKM: Reads per kilobase of transcript per million mapped reads; RRW: Repeated random walk; SNP: Single nucleotide polymorphism; TSS:

Transcription start site

\section{Acknowledgments}

The authors kindly acknowledge Leonie Chiara Martens and Martin Vingron for insightful discussions.

\section{Funding}

This work was supported by the German Research Foundation (Deutsche Forschungsgemeinschaft, DFG) [grant number MA 4454/3-1]. The funding body played no role in the design of the study, collection, analysis, and interpretation of the data and in writing the manuscript.

\section{Availability of data and materials}

The datasets used for the analysis presented in this article are either available in the https://github.molgen.mpg.de/denise/GIT_IncRNA_networks repository or downloadable via the scripts contained in the repository. The repository also contains most of the code used for the analysis. The Matlab code for the MSM clustering algorithm is available on request from the corresponding author, while the pseudocode is available from the corresponding publication at http://publications.imp.fu-berlin.de/1127/. Further data supporting the conclusions of this article, such as IncRNA-gene clusters and functional enrichment within clusters are provided as additional files.

\section{Authors' contributions}

Conceived the idea: AM. Designed the study: DT, NC, HS, AM. Developed the MSM clustering algorithm: NC. Implemented the methodology, performed the statistical modeling and analysis: DT. Contributed to the analysis of the results: RP, EN, HS. Wrote the paper: DT, NC, EN, HS, AM. All authors read and approved the final manuscript. 


\section{Ethics approval and consent to participate} Not applicable.

\section{Consent for publication \\ Not applicable.}

\section{Competing interests}

The authors declare that they have no competing interests.

\section{Publisher's Note}

Springer Nature remains neutral with regard to jurisdictional claims in published maps and institutional affiliations.

\section{Author details}

${ }^{1}$ Max Planck Institute for Molecular Genetics, Berlin, Ihnestraße 63-73, 14195 Berlin, Germany. ${ }^{2}$ Department of Mathematics and Informatics, Freie Universität, Berlin, Arnimallee 7, 14195 Berlin, Germany. ${ }^{3}$ Zuse Institute Berlin (ZIB), Takustraße 7, 14195 Berlin, Germany. ${ }^{4}$ Institute of Computational Biology (ICB), Helmholtz Zentrum München, Ingolstädter Landstraße 1, 85764 Oberschleißheim, Germany.

Received: 29 April 2019 Accepted: 13 May 2019

Published online: 29 May 2019

\section{References}

1. Yu AD, Wang Z, Morris KV. Long noncoding RNAs: a potent source of regulation in immunity and disease. Immunol Cell Biol. 2015;93(3): 277-283.

2. Ulitsky I, Bartel DP. lincrnas: genomics, evolution, and mechanisms. Cell. 2013;154(1):26-46.

3. Djebali S, David CS, Dobin A, Lassmann T, Mortazavi A. Landscape of transcription in human cells. Nature. 2012;489(7414):101-108.

4. Consortium EP. An integrated encyclopedia of DNA elements in the human genome. Nature. 2012;489(7414):57-74.

5. Amaral P, Clark MB, Gascoigne DK, Dinger ME, Mattick JS. Incrnadb: a reference database for long noncoding rnas. Nucleic Acids Res. 2011:39(Database issue):146-51.

6. Volders PJ, Helsens K, Wang X, Menten B, Martens L, Gevaert K, Vandesompele J, Mestdagh P. LNCipedia: a database for annotated human IncRNA transcript sequences and structures. Nucleic Acids Res. 2013:41(Database issue):246-51.

7. Jalali S, Kapoor S, Sivadas A, Bhartiya D, Scaria V. Computational approaches towards understanding human long non-coding rna biology. Bioinformatics. 2015:31(2241-51):14

8. Xie C, et al. NONCODEv4: exploring the world of long non-coding RNA genes. Nucleic Acids Research. 2014;42(D1):98-103.

9. Bassett AR, Akhtar A, Barlow DP, Bird AP, Brockdorff N. Considerations when investigating IncRNA function in vivo. Elife. 2014;14(3):03058.

10. Rinn JL, Chang HY. Genome regulation by long noncoding rnas. Annu Rev Biochem. 2012:81:145-66.

11. Morris KV, Mattick JS. The rise of regulatory rna. Nat Rev Genet. 2014;15(6):423-437.

12. Bulger $M$, Groudine $M$. Enhancers: the abundance and function of regulatory sequences beyond promoters. Dev Biol. 2010;339(2):250-257.

13. Orom UA, Derrien T, Beringer M, Gumireddy K, Gardini A, Bussotti G, Lai F, Zytnicki M, Notredame C, Huang Q, Guigo R, Shiekhattar R. Long noncoding rnas with enhancer-like function in human cells. Cell. 2010;143(1):46-58.

14. Lai F, Orom UA, Cesaroni M, Beringer M, Taatjes DJ, Blobel GA, Shiekhattar R. Activating rnas associate with mediator to enhance chromatin architecture and transcription. Nature. 2013;494(7438): 497-501.

15. Sandhu KS, Li G, Poh HM, Quek YL, Sia YY, Peh SQ, Mulawadi FH, Lim J, Sikic M, Menghi F, Thalamuthu A, Sung WK, Ruan X, Fullwood MJ, Liu E, Csermely $P$, Ruan Y. Large-scale functional organization of long-range chromatin interaction networks. Cell Rep. 2012;2(5):1207-19.

16. Fullwood MJ, Liu MH, Pan YF, Liu J, Xu H, Mohamed YB, Orlov Y. L. e.a. An oestrogen-receptor-alpha-bound human chromatin interactome. Nature. 2009;462(7269):58-64.

17. Liao Q, Liu C, Yuan X, Kang S, Miao R, Xiao H, Zhao G, Luo H, Bu D, Zhao H, Skogerbo G, Wu Z, Zhao Y. Large-scale prediction of long non-coding rna functions in a coding-non-coding gene co-expression network. Nucleic Acids Res. 2011;39(9):3864-78.

18. Perron U, Provero $P$, Molineris I. In silico prediction of IncRNA function using tissue specific and evolutionary conserved expression. BMC Bioinformatics. 2017;18(Suppl 5):144.

19. Spicuglia S, Maqbool MA, Puthier D, Andrau JC. An update on recent methods applied for deciphering the diversity of the noncoding rna genome structure and function. Methods. 2013;63(1):3-17.

20. Li G, Fullwood MJ, Xu H, Mulawadi FH, Velkov S, Vega V, Ariyaratne PN, Mohamed YB, Ooi HS, Tennakoon C, Wei CL, Ruan Y, Sung WK. An oestrogen-receptor-alpha-bound human chromatin interactome. Genome Biol. 2010;11(2):22.

21. Fotuhi SA, Ay F, Roy S. A multi-task graph-clustering approach for chromosome conformation capture data sets identifies conserved modules of chromosomal interactions. Genome Biol. 2016:17(1):114.

22. Albert R. Scale-free networks in cell biology. Journal of Cell Science. 2005:118(21):4947-4957.

23. Djurdjevac N, Bruckner S, Conrad TOF, Schütte C. Random walks on complex modular networks. Journal of Numerical Analysis, Industrial and Applied Mathematics. 2011;6:29-50

24. Sarich M, Conrad ND, Bruckner S, Conrad TOF, Schütte C. Modularity revisited: A novel dynamics-based concept for decomposing complex networks. Journal of Computational Dynamics. 2014;1(1):191-212.

25. Li G, Fullwood MJ, Xu H, Mulawadi FH, Velkov S, Vega V, Ariyaratne PN, Mohamed YB, Ooi HS, Tennakoon C, Wei CL, Ruan Y, Sung W. Chia-pet tool for comprehensive chromatin interaction analysis with paired-end tag sequencing. Genome Biol. 2010;11(2):22

26. Anders S, Pyl PT, Huber W. Htseq-a python framework to work with high-throughput sequencing data. Bioinformatics. 2014;31(2):166-9.

27. Ernst J, Kellis M. Chromhmm: automating chromatin-state discovery and characterization. Nat Methods. 2012;9(3):215-6.

28. Hamosh A, Scott AF, Amberger JS, Bocchini CA, McKusick VA. Online Mendelian Inheritance in Man (OMIM), a knowledgebase of human genes and genetic disorders. Nucleic Acids Res. 2005;33(Database issue):D514-7.

29. Pinero J, Bravo L, Queralt-Rosinach N, Gutiérrez-Sacristán A, Deu-Pons J, Centeno E, García-García J, Sanz F, Furlong LI. Disgenet: a comprehensive platform integrating information on human disease-associated genes and variants. Nucleic Acids Research. 2017;45(D1):833-839.

30. Chen G, Wang Z, Wang D, Qiu C, Liu M, Chen X, Zhang Q, Yan G, Cui Q. Lncrnadisease: a database for long-non-coding rna-associated diseases. Nucleic Acids Research. 2013:41(Database issue):983-6.

31. Amaral PP, Leonardi T, Han N, Viré E, Gascoigne DK, Arias-Carrasco R, Büscher M, Pandolfini L, Zhang A, Pluchino S, Maracaja-Coutinho V Nakaya HI, Hemberg M, Shiekhattar R, Enright AJ, Kouzarides T. Genomic positional conservation identifies topological anchor point RNAs linked to developmental loci. Genome Biol. 2018;19(1):32.

32. Hnisz D, Abraham BJ, Lee TI, Lau A, Saint-André V, Sigova AA, Hoke HA, Young RA. Super-enhancers in the control of cell identity and disease. Cell. 2013;155(4):934-47.

33. Liu S, Horlbeck M, Cho S, Birk H, Malatesta M, He D, Attenello F, Villalta J, Cho M, Chen Y, Mandegar M, Olvera M, Gilbert L, Conklin B, Chang H, Weissman J, Lim D. Crispri-based genome-scale identification of functional long noncoding rna loci in human cells. Science. 2017:355(6320):7111.

34. Newman M. Networks: An Introduction. New York: Oxford University Press, Inc.; 2010

35. Metzner P, Schütte C, Vanden-Eijnden E. Transition path theory for markov jump processes. Multiscale Modeling \& Simulation. 2009;7(3): 1192-1219.

36. Studholme C, Hill DLG, Hawkes DJ. An overlap invariant entropy measure of $3 \mathrm{~d}$ medical image alignment. Proc Medical Imaging. 1998:3338(1):132-143.

37. Csardi G, Nepusz T. The igraph software package for complex network research. InterJournal. 2006;Complex Systems:1695.

38. Clauset A, Newman MEJ, Moore C. Finding community structure in very large networks. Phys Rev E. 2004;70:066111.

39. Newman MEJ, Girvan M. Finding and evaluating community structure in networks. Phys Rev E. 2004;69:026113.

40. Newman MEJ. Finding community structure in networks using the eigenvectors of matrices. Phys Rev E. 2006;74:036104.

41. Pons $P$, Latapy M. Computing Communities in Large Networks Using Random Walks. JGAA. 2006;10(2):191-218. 
42. Morgan M, Falcon S, Gentleman R. GSEABase: Gene Set Enrichment Data Structures and Methods. 2017. R package version 1.40.1.

43. Lu Y, Shan G, Xue J, Chen C, Zhang C. Defining the multivalent functions of ctcf from chromatin state and three-dimensional chromatin interactions. Nucleic Acids Res. 2016;44(13):6200-12.

44. Ruan $W$, Wang $P$, Feng $S$, Xue Y, Li Y. Long non-coding rna small nucleolar rna host gene 12 (snhg12) promotes cell proliferation and migration by upregulating angiomotin gene expression in human osteosarcoma cells. Tumor Biol. 2016;37(3):4065-73.

45. Casero D, Sandoval S, Seet CS, Scholes J, Zhu Y, Ha VL, Luong A, Parekh C, Crooks GM. Long non-coding rna profiling of human lymphoid progenitor cells reveals transcriptional divergence of $\mathrm{b}$ cell and $\mathrm{t}$ cell lineages. Nat Immunol. 2015;16(12):1282-91.

46. West AH, Godley LA, Churpek JE. Familial myelodysplastic syndrome/acute leukemia syndromes: a review and utility for translational investigations. Ann N Y Acad Sci. 2014;1310:111-8.

47. Mogilyansky E, Rigoutsos I. The mir-17/92 cluster: a comprehensive update on its genomics, genetics, functions and increasingly important and numerous roles in health and disease. Cell Death Differ. 2013;20(12): 1603-14.

48. McHugh CA, Chen CK, Chow A, Surka CF, Tran C, McDonel P, Pandya-Jones A, Blanco M, Burghard C, Moradian A, Sweredoski MJ, Shishkin AA, Su J, Lander ES, Hess S, Plath K, Guttman M. The xist Incrna interacts directly with sharp to silence transcription through hdac3. Nature. 2015;521(7551):232-6.

49. Nobili L, Lionetti M, Neri A. Long non-coding rnas in normal and malignant hematopoiesis. Oncotarget. 2016;7(31):50666-50681.

50. Cai L, Chang H, Fang Y, Li G. A comprehensive characterization of the function of lincrnas in transcriptional regulation through long-range chromatin interactions. Sci Rep. 2016;6:36572. https://doi.org/10.1038/ srep36572.

51. Liu Y, Zhao J, Zhang W, Gan J, Hu C, Huang G, Zhang Y. Incrna gas5 enhances $\mathrm{g} 1$ cell cycle arrest via binding to ybx1 to regulate p21 expression in stomach cancer. Sci Rep. 2015;5:10159. https://doi.org/10. 1038/srep10159.

52. Muggen AF, Pillai SY, Kil LP, van Zelm MC, van Dongen JJ, Hendriks RW Langerak AW. Basal ca(2+) signaling is particularly increased in mutated chronic lymphocytic leukemia. Leukemia. 2015;29(2):321-8.

53. Wu L, et al. A transcriptome-wide association study of 229,000 women identifies new candidate susceptibility genes for breast cancer. Nat Genet. 2018;50(7):968-978.

\section{Ready to submit your research? Choose BMC and benefit from:}

- fast, convenient online submission

- thorough peer review by experienced researchers in your field

- rapid publication on acceptance

- support for research data, including large and complex data types

- gold Open Access which fosters wider collaboration and increased citations

- maximum visibility for your research: over $100 \mathrm{M}$ website views per yea

At $B M C$, research is always in progress.

Learn more biomedcentral.com/submission 